\title{
Assessment of methods for the numerical solution of the Fredholm integral eigenvalue problem
}

\author{
W. Betz, I. Papaioannou \& D. Straub \\ Engineering Risk Analysis Group, Technische Universität München, Germany
}

\begin{abstract}
The computational efficiency of random field representations with the Karhunen-Loève expansion relies on the numerical solution of a Fredholm integral eigenvalue problem. In this contribution, different methods for this task are compared. These include the finite element method (FEM), the finite cell method (FCM) and the Nyström method. For the FEM with linear basis functions, two different approaches to treat the covariance function in the integral eigenvalue problem are investigated: $L^{2}$-projection and linear interpolation of the covariance function between the nodes of the finite element mesh. The FCM is a novel approach, originally presented in (Parvizian et al., Comput Mech, 41: 121-133, 2007) for the solution of elliptic boundary value problems. This method is based on an extension to the FEM but avoids mesh generation on domains of complex geometric shape. In the Nyström method, a numerical integration rule is applied to transform the integral eigenvalue problem to a matrix eigenvalue problem. It is shown that the expansion optimal linear estimation (EOLE) method proposed in (Li \& Der Kiureghian, J Eng Mech-ASCE, 119(6): 1136-1154, 1993) constitutes a special case of the Nyström method. The behavior of all methods is investigated with respect to a two-dimensional example of a plate with a hole.
\end{abstract}

\section{INTRODUCTION}

Uncertain variables with inherent spatial variability, such as soil parameters in geotechnical engineering or material properties in civil engineering are modeled by means of random fields. A random field represents a random quantity at each point of a continuous domain, and, thus, consists of an infinite number of random variables. The approximation of the field by means of a finite number of random variables is referred to as random field discretization. This process is essential for a numerical representation of random fields. An overview of random field discretization methods can be found in Sudret \& Der Kiureghian (2000). Series expansion methods approximate the random field by a finite sum of products of deterministic spatial functions and random variables. Among series expansion methods, the Karhunen-Loève (KL) expansion is of special interest. This is because it can approximate the original random field accurately with a minimum number of random variables (Ghanem \& Spanos 1991).

The KL expansion requires the solution of a Fredholm integral eigenvalue problem (IEVP), whose integral kernel is the autocovariance function of the random field. Analytical solutions of the IEVP are available only for a few autocovariance functions and for simple geometries. Therefore, for most practical problems, the IEVP must be solved numerically. An overview on the numerical solution of Fredholm integral equations is found in (Atkinson 1997). Ghanem and Spanos (1991) proposed to solve the problem by means of the finite element method (FEM) that is based on the Galerkin procedure. On the other hand, the Nyström method approaches the problem through a quadrature representation of the integral equation. Alternative methods for solving the IEVP are the collocation method and the degenerate kernel method (Atkinson 1997).

In this study, the FEM and the Nyström method are assessed. Besides the standard FEM, an alternative formulation where the shape functions are projected onto the space spanned by the linear basis functions (Keese 2004) is considered. Furthermore, a novel approach, termed finite cell method (FCM), is proposed. The FCM can be regarded as an extension to the FEM, but does not require an explicit meshing of the domain of the random field. The method was originally proposed in (Parvizian et al. 2007) for the solution of elliptic boundary value problems. In the FCM, the domain of computation is extended beyond the physical domain up to the boundaries of an embedding domain with a primitive geometrical shape. Advanced integration techniques are required to cope with the discontinuity appearing at the boundary of the physical domain. 
The methods are presented in detail and their performance is assessed with the help of a twodimensional numerical example. We focus on the convergence behavior of the methods and the computational efforts needed to approximate the random field.

NOTE: This is a shortened version of Betz et al. (2013).

\section{KARHUNEN-LOEVE EXPANSION}

\subsection{Definition of random fields}

A continuous random field $H(\mathbf{x}, \theta)$ may be loosely defined as a random function that describes a random quantity at each point $\mathbf{x} \in \Omega$ of a continuous domain $\Omega \subset \mathbb{R}^{d}, d \in \mathbb{N}_{>0} . \theta \in \Theta$ is a coordinate in the sample space $\Theta$, and $(\Theta, F, P)$ is a complete probability space. If the random quantity attached to each point $\mathbf{x}$ is a random variable, the random field is said to be univariate or real-valued. If the random quantity is a random vector, the field is called multivariate. The dimension $d$ of a random field is the dimension of its topological space $\Omega$. One usually distinguishes between a one- and a multidimensional random field.

The field is said to be Gaussian if the distribution of $\left(H\left(\mathbf{x}_{1}, \theta\right), \ldots, H\left(\mathbf{x}_{n}, \theta\right)\right)$ is jointly Gaussian for any $\left(\mathbf{x}_{1}, \ldots, \mathbf{x}_{n}\right) \in \Omega$ and any $n \in \mathbb{N}_{>0}$. A Gaussian field is completely defined by its mean function $\mu: \Omega \rightarrow \mathbb{R}$ and autocovariance function Cov : $\Omega \times \Omega \rightarrow \mathbb{R}$. The autocovariance function can be expressed as $\operatorname{Cov}\left(\mathbf{x}, \mathbf{x}^{\prime}\right)=\sigma(\mathbf{x}) \cdot \sigma\left(\mathbf{x}^{\prime}\right) \cdot \rho\left(\mathbf{x}, \mathbf{x}^{\prime}\right)$, where $\sigma: \Omega \rightarrow \mathbb{R}$ is the standard deviation function of the random field and $\rho: \Omega \times \Omega \rightarrow[-1 ; 1]$ is its autocorrelation coefficient function.

The discussion in this work is restricted to univariate multidimensional Gaussian random fields. Some non-Gaussian random fields, namely the so-called translation fields (Grigoriu 1984), can be expressed by means of Gaussian random fields through a nonlinear mapping.

\subsection{KL expansion of Gaussian random fields}

The Karhunen-Loève expansion is a series expansion method for the representation of the random field. The expansion is based on a spectral decomposition of the autocovariance function of the field. It states that a random field can be represented exactly by the following expansion (Loève 1977, Van Trees 1968):

$H(\mathbf{x}, \theta)=\mu(\mathbf{x})+\sum_{i=1}^{\infty} \sqrt{\lambda_{i}} \varphi_{i}(\mathbf{x}) \xi_{i}(\theta)$

where $\mu(\mathbf{x})$ is the mean function of the field, $\xi_{i}(\theta)$ : $\Theta \rightarrow \mathbb{R}$ are independent standard normal random variables, and $\lambda_{i} \in[0, \infty), \varphi_{i}: \Omega \rightarrow \mathbb{R}$ are the eigenvalues and eigenfunctions of the autocovariance kernel obtained from solving the homogeneous Fredholm integral equation of the second kind:

$\int_{\Omega} \operatorname{Cov}\left(\mathbf{x}, \mathbf{x}^{\prime}\right) \varphi_{i}\left(\mathbf{x}^{\prime}\right) \mathrm{d} \mathbf{x}^{\prime}=\lambda_{i} \varphi_{i}(\mathbf{x})$

The eigenfunctions are by definition orthonormal, i.e. $\int_{\Omega} \varphi_{i}(\mathbf{x}) \varphi_{j}(\mathbf{x}) \mathrm{d} \mathbf{x}=\delta_{i j}$, where $\delta_{i j}$ is one if $i=j$ and zero otherwise. Moreover, they form a complete basis of the space $L^{2}(\Omega)$ of square integrable functions on $\Omega$.

\subsection{Truncated KL expansion}

The truncated KL expansion is obtained by arranging the eigenvalues and eigenfunctions in a descending series with respect to the magnitude of the eigenvalues, and truncating the ordered expansion after $M$ terms. The truncated KL expansion does no longer represent the random field $H(\cdot)$ exactly, but provides an approximation $\tilde{H}(\cdot)$ of the field. Hence, the truncated KL expansion is a random field discretization method. The discretized random field is written as:

$\tilde{H}(\mathbf{x}, \theta)=\mu(\mathbf{x})+\sum_{i=1}^{M} \sqrt{\lambda_{i}} \varphi_{i}(\mathbf{x}) \xi_{i}(\theta)$

For fixed $M$, the resulting random field approximation $\tilde{H}(\mathbf{x}, \theta)$ is optimal among series expansion methods with respect to the integral of the mean square truncation error over the domain $\Omega$, given any other complete basis of $L^{2}(\Omega)$ (Ghanem \& Spanos 1991).

\section{NUMERICAL METHODS}

Integral eigenvalue problems of the type given in Equation (2) are difficult to solve analytically except for a few autocovariance functions defined on domains $\Omega$ of simple geometric shape. In general, the integral eigenvalue problem is solved numerically. Thus, the random field approximation of the truncated $\mathrm{KL}$ expansion given in Equation (3) is approximated as:

$$
\hat{H}(\mathbf{x}, \theta)=\mu(\mathbf{x})+\sum_{i=1}^{M} \sqrt{\hat{\lambda}_{i}} \hat{\varphi}_{i}(\mathbf{x}) \hat{\xi}_{i}(\theta)
$$

where $\hat{\lambda}_{i}$ and $\hat{\varphi}_{i}$ are approximations to the true eigenvalues $\lambda_{i}$ and eigenfunctions $\varphi_{i} . \hat{\xi}_{i}(\theta)$ are independent standard normal random variables. Numerical methods for the solution of Fredholm integral eigenvalue problems approximate the eigenfunctions by a set of functions $h_{j}: \Omega \rightarrow \mathbb{R}$ as:

$\varphi_{i}(\mathbf{x}) \approx \hat{\varphi}_{i}(\mathbf{x})=\sum_{j=1}^{N} d_{j}^{i} h_{j}(\mathbf{x})$

where the coefficients $d_{j}^{i} \in \mathbb{R}$ have to be determined. 


\subsection{Nyström method}

In the Nyström method (Atkinson 1997), the integral in the eigenvalue problem of Equation (2) is approximated by a numerical integration scheme:

$\sum_{j=1}^{N} w_{j} \operatorname{Cov}\left(\mathbf{x}, \mathbf{x}_{j}\right) \hat{\varphi}_{i}\left(\mathbf{x}_{j}\right)=\hat{\lambda}_{i} \hat{\varphi}_{i}(\mathbf{x})$

where $\mathbf{x}_{j} \in \Omega$ with $j \in\{1, \ldots, N\}, N \in \mathbb{N}$, represent a finite set of integration points, and $w_{j}$ is the integration weight associated with each $\mathbf{x}_{j}$. The Nyström method solves Equation (6) at the integration points, i.e.:

$\sum_{j=1}^{N} w_{j} \operatorname{Cov}\left(\mathbf{x}_{k}, \mathbf{x}_{j}\right) \hat{\varphi}_{i}\left(\mathbf{x}_{j}\right)=\hat{\lambda}_{i} \hat{\varphi}_{i}\left(\mathbf{x}_{k}\right), \quad k=1, \ldots, N$

The equation above can be formulated in matrix notation as

$\mathbf{C W y}_{i}=\hat{\lambda}_{i} \mathbf{y}_{i}$

where $\mathbf{C}$ is a symmetric positive semi-definite $N \times N$ matrix with elements $C_{k j}=\operatorname{Cov}\left(\mathbf{x}_{k}, \mathbf{x}_{j}\right), \mathbf{W}$ is a diagonal matrix of size $N$ with nonnegative diagonal entries $W_{j j}=w_{j}$, and $\mathbf{y}_{i}$ is a $N$-dimensional vector whose $k$ th entry is $y_{i, k}=\hat{\varphi}_{i}\left(\mathbf{x}_{k}\right)$. From a numerical point of view, it is advantageous to reformulate the matrix eigenvalue problem in Equation (8) to the equivalent matrix eigenvalue problem $\mathbf{B} \mathbf{y}_{i}^{*}=\hat{\lambda}_{i} \mathbf{y}_{i}^{*}$, where $\mathbf{B}$ is a symmetric positive semi-definite matrix defined as $\mathbf{B}=\mathbf{W}^{\frac{1}{2}} \mathbf{C} \mathbf{W}^{\frac{1}{2}}$, and $\mathbf{W}^{\frac{1}{2}}$ is a diagonal matrix with diagonal entries $\sqrt{w_{j}}$. The eigenvectors $\mathbf{y}_{i}$ can be computed as $\mathbf{y}_{i}=\mathbf{W}^{-\frac{1}{2}} \mathbf{y}_{i}^{*}$, where $\mathbf{W}^{-\frac{1}{2}}$ denotes the inverse of the matrix $\mathbf{W}^{\frac{1}{2}}$.

The eigenfunctions are obtained by means of the interpolation

$\hat{\varphi}_{i}(\mathbf{x})=\frac{1}{\hat{\lambda}_{i}} \sum_{j=1}^{N} \sqrt{w_{j}} y_{i, j}^{*} \operatorname{Cov}\left(\mathbf{x}, \mathbf{x}_{j}\right)$

where $y_{i, j}^{*}$ is the $j$ th element of the eigenvector $\mathbf{y}_{i}^{*}$. Equation 9 is derived by solving equation 6 for $\hat{\varphi}_{i}(\mathbf{x})$. It should be noted that a normalization of the eigenvectors $\left\|\mathbf{y}_{i}^{*}\right\|=1$ is equivalent to a normalization of the eigenfunctions.

\subsection{Equivalence of the EOLE method with the Nyström method}

If all the integration weights $w_{j}$ in the numerical integration scheme applied in the Nyström method are the same, i.e. $w_{j}=w, \forall j=1, \ldots, N$, the matrix $\mathbf{W}$ in
Equation (8) can be written as $\mathbf{W}=w \mathbf{I}$, where $\mathbf{I}$ is the identity matrix and $w=|\Omega| / N$. In this special case, the matrix eigenvalue problem of Equation (8) can be reformulated as:

$\mathbf{C y}_{i}=\hat{\lambda}_{i}^{*} \mathbf{y}_{i}$

where $\hat{\lambda}_{i}^{*}$ is related to $\hat{\lambda}_{i}$ in Equation (8) as $\hat{\lambda}_{i}^{*}=\frac{N}{|\Omega|} \hat{\lambda}_{i}$. For this special case, $\hat{\lambda}_{i}^{*}$ and $\mathbf{y}_{i}$ are the eigenvalues and eigenfunctions of the covariance matrix $\mathbf{C}$. Assuming normalized eigenvectors $\mathbf{y}_{i}$, i.e. $\left\|\mathbf{y}_{i}\right\|=1$, gives, after some algebra, the following approximate truncated KL expansion:

$\hat{H}(\mathbf{x}, \theta)=\mu(\mathbf{x})+\sum_{i=1}^{M} \frac{\hat{\xi}_{i}(\theta)}{\sqrt{\hat{\lambda}_{i}^{*}}} \sum_{j=1}^{N} y_{i, j} \operatorname{Cov}\left(\mathbf{x}, \mathbf{x}_{j}\right)$

where $y_{i, j}$ is the $j$ th element of $\mathbf{y}_{i}$. Comparing Equation (10) and Equation (11) with the equations of the expansion optimal linear estimation (EOLE) method, proposed in (Li \& Der Kiureghian 1993), shows that the same problem as in the EOLE method is solved. Therefore, the EOLE method with a uniform distribution of points over the domain can be considered a special case of the Nyström method with equal integration weights. Consequently, the EOLE method is an approximation to the solution of the KarhunenLoève expansion.

\subsection{Galerkin methods}

\subsubsection{Finite element method}

In order to quantify the error due to the approximation of the eigenfunctions in Equation (5), the residual $r_{\mathrm{IEVP}}(\mathbf{x})$ is introduced as:

$r_{\mathrm{IEVP}}(\mathbf{x})=\sum_{j=1}^{N} d_{j}^{i}\left(\int_{\Omega} \operatorname{Cov}\left(\mathbf{x}, \mathbf{x}^{\prime}\right) h_{j}\left(\mathbf{x}^{\prime}\right) \mathrm{d} \mathbf{x}^{\prime}-\hat{\lambda}_{i} h_{j}(\mathbf{x})\right)$

In general, the coefficients $d_{j}^{i}$ are selected such that the residual $r_{\text {IEVP }}$ is minimized in some sense. Different techniques on how to minimize $r_{\text {IEVP }}$ exist. A point-wise minimization of $r_{\text {IEVP }}$ is utilized by the socalled collocation methods (Atkinson 1997). For the Galerkin methods, the coefficients $d_{j}^{i}$ are chosen such that the residual $r_{\text {IEVP }}$ becomes orthogonal to the subspace spanned by the basis functions, i.e.:

$\int_{\Omega} r_{\mathrm{IEVP}}(\mathbf{x}) h_{k}(\mathbf{x}) \mathrm{d} \mathbf{x}=0 \quad \forall k=1, \ldots, N$

Equation 13 can be expressed in matrix notation by the following generalized matrix eigenvalue problem (Ghanem \& Spanos 1991)

$\mathbf{B d}_{i}=\hat{\lambda}_{i} \mathbf{M d}_{i}$ 


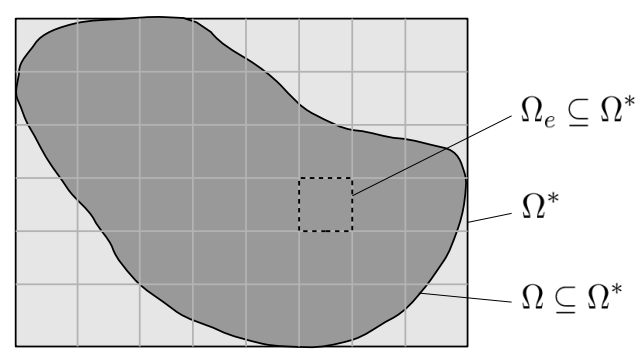

Figure 1: The finite cell method - idea and notation.

where $\mathbf{B}$ is a symmetric positive semi-definite $N \times N$ matrix whose elements are defined as

$B_{l m}=\int_{\Omega} h_{l}(\mathbf{x}) \int_{\Omega} \operatorname{Cov}\left(\mathbf{x}, \mathbf{x}^{\prime}\right) h_{m}\left(\mathbf{x}^{\prime}\right) \mathrm{d} \mathbf{x}^{\prime} \mathrm{d} \mathbf{x}$

and $\mathbf{M}$ is a symmetric positive definite $N \times N$ matrix with elements

$M_{l m}=\int_{\Omega} h_{l}(\mathbf{x}) h_{m}(\mathbf{x}) \mathrm{d} \mathbf{x}$

If the domain $\Omega$ is of simple geometric shape, the basis functions can be selected such that they are defined globally on the domain $\Omega$. However, in practical problems the domain $\Omega$ has often a complex geometrical shape. In this case, $\Omega$ can be approximated by a discretization into multiple elements. The basis functions are then defined locally on the element domains. This approach is called finite element method (FEM). In the FEM, the basis functions are usually chosen as piecewise linear polynomials. However, it is noted that the use of higher-order basis functions can greatly improve the convergence behavior of the method.

Instead of solving Equation (14) directly, the covariance function can first be projected onto the space spanned by the basis functions $\left\{h_{j}\right\}_{j=1}^{N}$, i.e.:

$\operatorname{Cov}\left(\mathbf{x}, \mathbf{x}^{\prime}\right) \approx \sum_{j=1}^{N} \sum_{k=1}^{N} K_{j k} h_{j}(\mathbf{x}) h_{k}\left(\mathbf{x}^{\prime}\right)$

Inserting Equation (17) into Equation (15) leads to $\mathbf{B}=\mathbf{M K M}$, where the matrix $\mathbf{K}$ is a symmetric positive semi-definite $N \times N$ matrix with coefficients $K_{j k}$. The coefficients $K_{j k}$ have to be determined beforehand. If only piecewise linear basis functions are applied, the coefficients can be computed as $K_{j k}=\operatorname{Cov}\left(\mathbf{x}_{j}, \mathbf{x}_{k}\right)$, where $\mathbf{x}_{j}$ and $\mathbf{x}_{k}$ denote the coordinates of the nodes of the FE mesh. This approach is referred to as linear projection. It should be noted that the solution of Equation (14) where $\mathbf{B}$ is assembled according to Equation (15) is equivalent to a $L^{2}$ projection.

\subsubsection{Finite cell method}

If a finite element mesh is not readily available, a meshless approach might be favored. One quasi meshless approach is based on so-called finite cells.

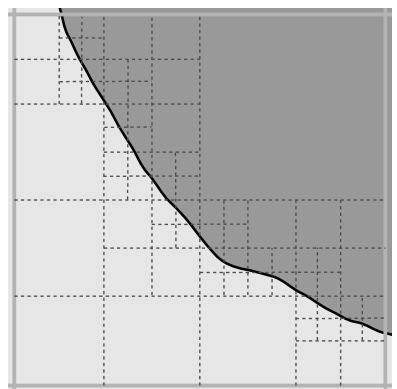

Figure 2: Staggered Gaussian integration: mesh for integration on a finite cell cut by $\partial \Omega$.

The finite cell method (FCM) (Parvizian et al. 2007) was originally developed as an extension to the FEM for the solution of linear elasticity problems. Let $\Omega \subset$ $\mathbb{R}^{d}$ be the domain of interest and $\Omega^{*} \subset \mathbb{R}^{d}$ a geometrically simpler domain with $\Omega \subset \Omega^{*}$. The geometrically simpler domain $\Omega^{*}$ is called primitive domain, and the original domain $\Omega$ is referred to as physical domain. In the FCM, not the physical but the primitive domain is meshed, and, therefore, mesh generation is a trivial task. The finite cell approach is illustrated in Figure 1.

Let the basis functions $h_{j}^{*}(\mathbf{x}) \in L^{2}\left(\Omega^{*}\right)$ form a basis of a subspace in $L^{2}\left(\Omega^{*}\right)$. In this regard, the approximation of the eigenfunctions given in Equation (5) is redefined to

$\varphi_{i}(\mathbf{x}) \approx \hat{\varphi}_{i}(\mathbf{x})=\sum_{j=1}^{N} d_{j}^{i} h_{j}^{*}(\mathbf{x})$

This means, the solution of the integral equation defined on the physical domain $\Omega$ is approximated with basis functions spanned over the primitive domain $\Omega^{*}$. Consequently, the integrals in Equation (15) and Equation (16) can be rewritten as integrals over the primitive domain $\Omega^{*}$ :

$$
B_{l m}=\int_{\Omega^{*}} \alpha(\mathbf{x}) h_{l}(\mathbf{x}) \int_{\Omega^{*}} \operatorname{Cov}\left(\mathbf{x}, \mathbf{x}^{\prime}\right) h_{m}\left(\mathbf{x}^{\prime}\right) \alpha\left(\mathbf{x}^{\prime}\right) \mathrm{d} \mathbf{x}^{\prime} \mathrm{d} \mathbf{x}
$$

$M_{l m}=\int_{\Omega^{*}} \alpha(\mathbf{x}) h_{l}(\mathbf{x}) h_{m}(\mathbf{x}) \mathrm{d} \mathbf{x}$

where the mapping $\alpha: \Omega^{*} \rightarrow\{0,1\}$ is one for $\mathbf{x} \in \Omega$ and zero otherwise. Note that the eigenfunctions have to be normalized on $\Omega$, i.e. $\int_{\Omega^{*}} \alpha(\mathbf{x}) \varphi_{i}(\mathbf{x})^{2} \mathrm{~d} \mathbf{x}=1$. Moreover, higher-order basis functions are of crucial importance for the applicability of the method because they yield a fast rate of convergence (Parvizian et al. 2007).

The integrals in Equation (19) and Equation (20) are smooth over the domain $\Omega$ but not even continuous over the domain $\Omega^{*}$. Therefore, it is important to use appropriate numerical integration schemes in order to keep the integration error small. Standard Gaussian quadrature does not work well for this type of problem since in this case the integrand cannot be approximated well by a polynomial function. Thus, it is suggested to apply a so-called staggered Gaussian integration scheme, proposed in (Düster et al. 2008). The 
idea of this technique is demonstrated in Figure 2 for a finite cell that is cut by the boundary of the physical domain $\Omega$, denoted $\partial \Omega$. The finite cell is sub-divided into smaller sub-cells using a tree-based refinement structure. The vicinity of the discontinuity is modeled with a higher resolution, by sub-dividing cells that are cut by $\partial \Omega$. Standard Gaussian integration is applied on the leaf sub-cells.

Some kernels $\operatorname{Cov}\left(\mathbf{x}, \mathbf{x}^{\prime}\right)$ are non-differentiable on the diagonal, i.e. at $\mathbf{x}=\mathbf{x}^{\prime}$. In this case, the integrand of the inner integral in Equation (19) is nondifferentiable for $\mathbf{x}=\mathbf{x}^{\prime}$, additionally to the discontinuity at $\partial \Omega$. Moreover, Equation (15) of the FEM is affected as well. This additional difficulty can be dealt with by a slight modification of the integration scheme described above: Instead of refining only the region around $\partial \Omega$ with a high resolution, the entire cell (or element) domain is refined with at least a predefined minimum resolution. This is done only if both $\mathbf{x}$ and $\mathbf{x}^{\prime}$ are located in the same cell (or element). Note that for the FEM, the loss in efficiency due to integration of non-differentiable kernels is almost negligible. This is because the integration scheme needs to be modified only for the part in the integral where $\mathbf{x}$ and $\mathbf{x}^{\prime}$ are located in the same element.

\section{NUMERICAL STUDY}

\subsection{Error measure}

One error measure often used in conjunction with the $\mathrm{KL}$ expansion is the mean normalized error variance $\varepsilon_{\sigma}$ :

$\varepsilon_{\sigma}=\frac{1}{|\Omega|} \int_{\Omega} \frac{\operatorname{Var}[H(\mathbf{x}, \theta)-\hat{H}(\mathbf{x}, \theta)]}{\operatorname{Var}[H(\mathbf{x}, \theta)]} \mathrm{d} \mathbf{x}$

The evaluation of this error measure is straightforward if we can write $\operatorname{Var}[H(\mathbf{x}, \theta)-\hat{H}(\mathbf{x}, \theta)]=$ $\operatorname{Var}[H(\mathbf{x}, \theta)]-\operatorname{Var}[\hat{H}(\mathbf{x}, \theta)]$. However, if the KL expansion is approximated numerically the above does not necessary hold. Therefore, in this study a different error measure is applied.

Let $\varepsilon_{\mathrm{Var}}$ be an error measure for the quantification of the quality of a random field discretization, defined as

$\varepsilon_{\mathrm{Var}}=\frac{1}{|\Omega|} \int_{\Omega} \frac{|\operatorname{Var}[H(\mathbf{x}, \theta)]-\operatorname{Var}[\hat{H}(\mathbf{x}, \theta)]|}{\operatorname{Var}[H(\mathbf{x}, \theta)]} \mathrm{d} \mathbf{x}$

For a fixed number $M$ of terms in the expansion of the random field, let $\varepsilon_{\text {Var,ref }}$ denote the reference error obtained with the expansion based on the true eigenvalues and eigenfunctions [Eq. (3)]. The presented methods for the solution of the KL expansion are investigated with respect to their convergence in the relative

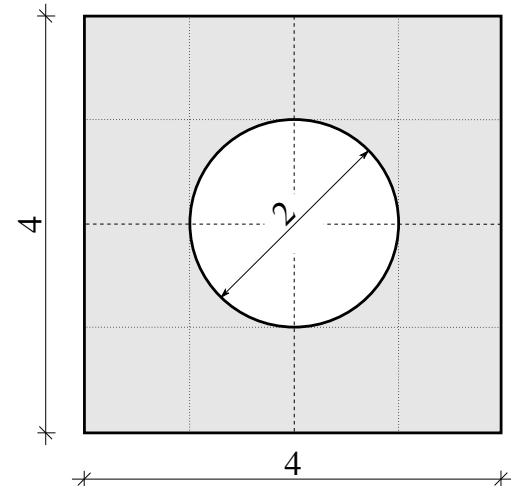

Figure 3: Shape of the domain used in the 2D example

error $\varepsilon_{\text {Var,rel }}$ :

$\varepsilon_{\mathrm{Var}, \mathrm{rel}}=\frac{\left|\varepsilon_{\mathrm{Var}}-\varepsilon_{\mathrm{Var}, \mathrm{ref}}\right|}{\varepsilon_{\mathrm{Var}, \mathrm{ref}}}$

\subsection{D example - exponential kernel}

The random field is modeled on a rectangular domain with a hole (Fig. 3), and has a constant standard deviation $\sigma=6 \cdot 10^{3}$. The number of random variables in the expansion is fixed to $M=30$. An autocorrelation coefficient function of the exponential type is selected:

$\rho\left(\left|\mathbf{x}-\mathbf{x}^{\prime}\right|\right)=\exp \left(-\frac{\left|\mathbf{x}-\mathbf{x}^{\prime}\right|}{l}\right)$

where $l$ denotes the correlation length. The correlation length is chosen such that the reference error is close to $5 \%$ (i.e. $l=4.2$ ). The exponential correlation structure defined in Equation (24) is non-differentiable at the diagonal, i.e. at $\mathbf{x}=\mathbf{x}^{\prime}$. As is mentioned in section 3.3.2, this introduces yet another difficulty in the integration of Equation (15) and Equation (19).

For the linear FEM, 4-node quadrilateral elements are applied to mesh the physical domain $\Omega$. The more elements are used the better $\Omega$ is represented by the mesh. The convergence behavior of the random field discretization is investigated with respect to an increasing number of elements. Additional to the conventional $L^{2}$-projection, linear projection of the covariance kernel is applied as well.

In the FCM, the physical domain $\Omega$ is embedded in a so-called primitive domain $\Omega^{*}$ of simple geometric shape. In this example a rectangular shape with the same contour as the rectangle in Figure 3 is chosen for $\Omega^{*}$, i.e. the circular hole of the physical domain is not considered by $\Omega^{*}$. Three different structured meshes are studied for the discretization of $\Omega^{*}: 1 \times 1,2 \times 2$ and $4 \times 4$ cells (Fig. 3 ). For each structured mesh, the convergence behavior of the random field discretization is investigated with respect to an increasing polynomial order of the basis functions of the cells.

The Nyström method with equal integration weights, i.e. the EOLE method, can be considered a truly meshless method. In this study, the integration 


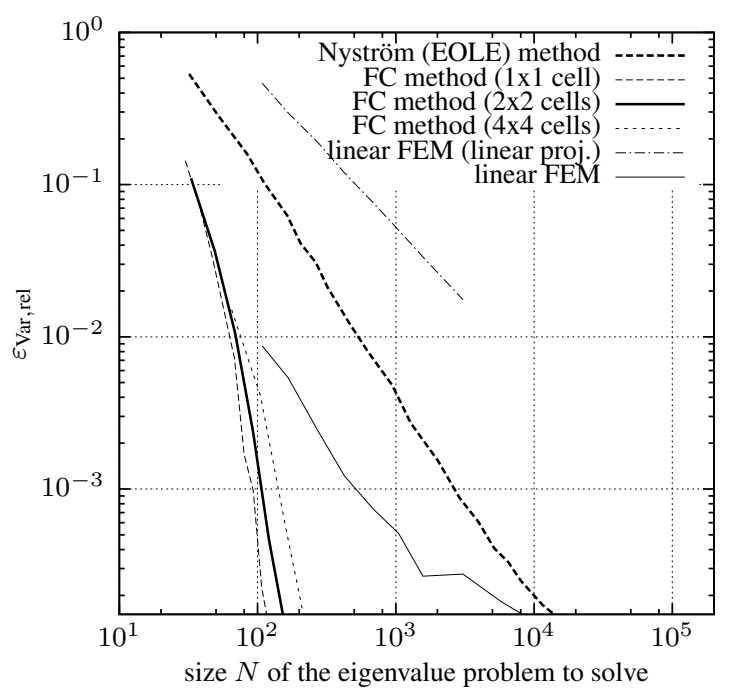

Figure 4: Convergence of the relative error $\varepsilon_{\mathrm{Var}, \text { rel }}$ with respect to the size of the matrix eigenvalue problem to solve. (exponential kernel)

points are distributed uniformly over the primitive domain $\Omega^{*}$ of simple geometric shape. Points located outside of the physical domain $\Omega$ are not considered in the analysis. The convergence behavior of the random field discretization is investigated with respect to an increasing number of points.

For each of the random field discretization methods, a matrix eigenvalue problem must be solved. In a first study, the convergence behavior of the relative error $\varepsilon_{\text {Var,rel }}$ is investigated with respect to the size of the matrix eigenvalue problem. The reference error $\varepsilon_{\text {Var,ref }}$ was obtained numerically as 0.049954 using the FCM with $4 \times 4$ cells and a maximum polynomial order of 20. Special care was taken to ensure a good quality of the numerical integrals involved. The results of the analysis are depicted in Figure 4 . The finite cell discretization scheme exhibits an exponential rate of convergence. The influence of the coarseness of the finite cell mesh is small. A smaller number of cells results in a faster convergence. However, to reach the same relative error, a larger maximum polynomial order is required on a coarser mesh. Contrary to the exponential rate of convergence of the FCM, the Nyström (EOLE) method and the linear FEM show only a linear rate of convergence in the log-log plot in Figure 4. The difference between the linear FEM and the FCM can be explained by the use of higherorder basis functions in the FCM. Linear-projection of the covariance kernel on the piecewise linear finite element basis functions converges much slower than the corresponding $L^{2}$-projection. Since the size of the matrix eigenvalue problem can become rather large (i.e. $N \gg M$ ) for the FEM and the Nyström method, it is usually much faster to compute only the $M$ largest eigenvalues and corresponding eigenvectors instead of finding all the $N$ eigenvalues and eigenvectors. This can be achieved by means of Lanczos algorithms for the solution of the matrix eigenvalue problem.

The size of the matrix eigenvalue problem to solve

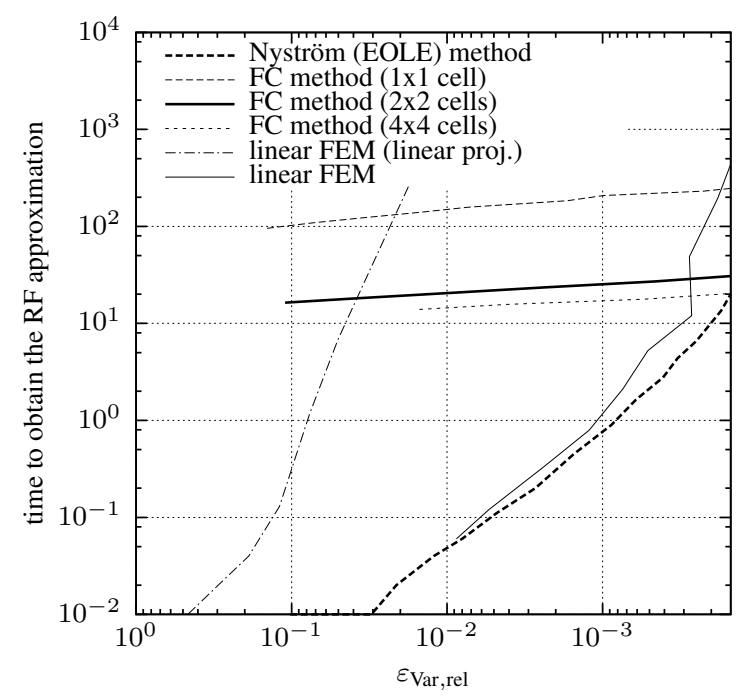

Figure 5: Time needed to obtain an approximation of a random field for a certain relative error $\varepsilon_{\text {Var,rel. }}$ (exponential kernel)

is only one property of the overall solution process. Another factor that might have considerable influence on the overall computational cost is the assembly of the matrices, which differs significantly between the different methods. Therefore, for practical purposes, a comparison of the computational time needed to obtain a solution of the random field approximation is of interest. This time is plotted in Figure 5 with respect to the relative error $\varepsilon_{\mathrm{Var}, \text { rel }}$ of the corresponding random field approximation. This study was performed on a Intel ${ }^{\circledR}$ Core $^{\mathrm{TM}}$ i7-3770 running at $3.40 \mathrm{GHz}$. All methods were implemented in $\mathrm{C}++$. Some effort was put into optimizing the implementations. In this regard, the Nyström (EOLE) method is the most efficient one. The FEM behaves slightly worse than the EOLE method. The difference between the two methods grows larger for decreasing relative errors. Except for very small errors, the linear FEM is clearly faster than the FCM. This is due to the time needed for integration of discontinuous functions in the FCM. In Figure 5, the curves corresponding to the FCM exhibit a flatter slope than the one corresponding to the FEM. This is caused by the use of higher-order basis functions in the FCM that lead to an exponential rate of convergence. Contrary to the findings of Figure 4, it is better to use more than a single cell. The relatively large difference observed between using a single cell and using four cells in the FCM, is due to a more efficient numerical treatment of the integrals in the later case (compare section 3.3.2). Compared to the other investigated methods, the FEM with linear projection of the covariance kernel using linear basis functions is very inefficient and, therefore, not of interest for practical applications.

If the random field is required as input for a nonintrusive finite element reliability analysis (Papaioannou 2013), for each run of the finite element method a realization of the random field needs to be evaluated at every Gauss-point. For this type of problem, a significant part of the overall runtime is spend in the evaluation of a realization of the random field. Therefore, 


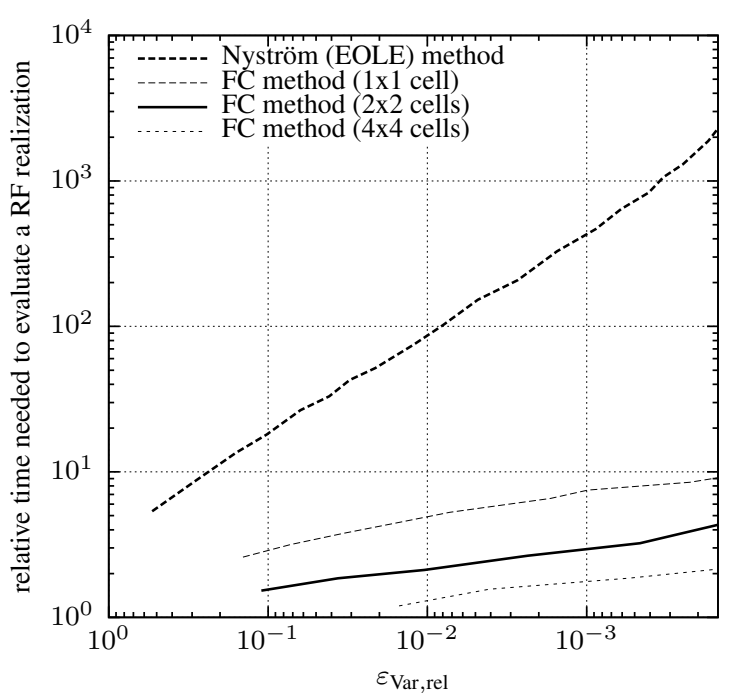

Figure 6: Relative time needed to evaluate a realization of the random field for a certain relative error $\varepsilon_{V a r, \text { rel }}$. The results are relative to $t_{\mathrm{hFEM}}$.

it is relevant to compare the methods with respect to the time needed to evaluate a single realization of the random field. In this regard, no other method can be more efficient than the linear FEM. This is because independent of the accuracy of the random field approximation, the number of basis functions to evaluate for a realization of the field remains constant: for a one, two- and three-dimensional element two, four and eight basis functions must be evaluated, respectively. On a Intel ${ }^{\circledR}$ Core $^{\mathrm{TM}}$ i $7-3770$ running at $3.40 \mathrm{GHz}$ this time is approximately $t_{\mathrm{hFEM}}=3.3 \cdot 10^{-7} \mathrm{~s}$. For all other methods, the time needed to obtain a realization was weighted with $t_{\mathrm{hFEM}}$. The results are presented in Figure 6. The computational costs of both the FCM and the Nyström method increase with a decreasing relative error $\varepsilon_{\text {Var,rel }}$. However, the Nyström (EOLE) method is more than an order of magnitude slower than the FCM. This is due to the fact that in the Nyström method the autocorrelation coefficient functions must be computed at every integration point, whereas in the FCM only the the basis functions local to the cell must be evaluated.

Therefore, if the time spend in the evaluation of random field realizations has a major contribution to the overall runtime, the Nyström (EOLE) method is not the best choice for random field discretization. If a finite element mesh is already available (e.g. as in non-intrusive finite element reliability analysis), the use of the finite element method for the discretization of the random field is recommended. In this case the mesh used for the finite element reliability analysis should be used for the random field discretization as well. For very small correlation lengths, the existing finite element mesh might be too coarse to approximate the solution of the IEVP well with linear basis functions. In this case higher-order basis functions can be used to improve the quality of the random field approximation. The use of higher-order basis functions is also recommended to check if the approximation has already converged with sufficient accuracy.

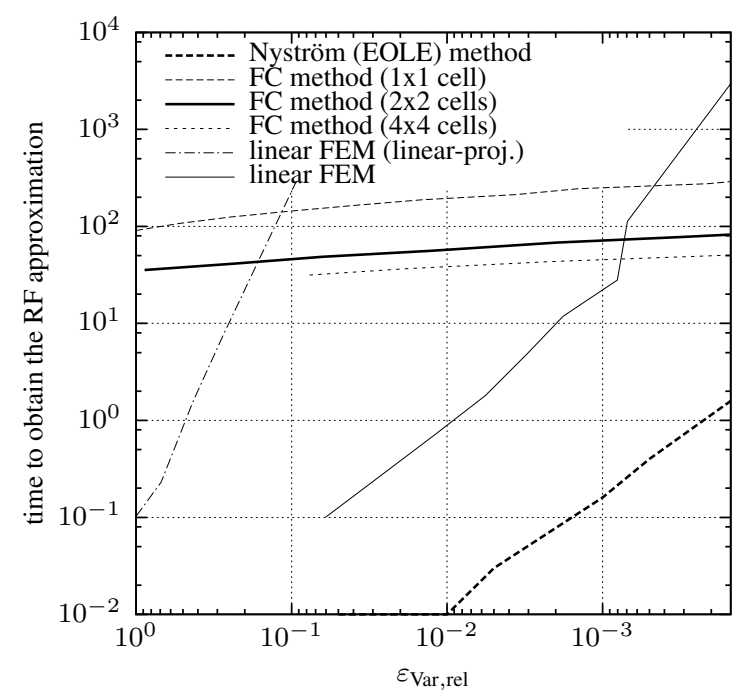

Figure 7: Time needed to obtain an approximation of a random field for a certain relative error $\varepsilon_{\text {Var,rel. }}$ (Gaussian kernel)

If a mesh is not available, the FCM is the method of choice.

\subsection{D example - Gaussian kernel}

The same problem as in section 4.2 is investigated, but replacing the non-differentiable exponential kernel (24) with the differentiable Gaussian kernel:

$\rho\left(\left|\mathbf{x}-\mathbf{x}^{\prime}\right|\right)=\exp \left[-\left(\frac{\left|\mathbf{x}-\mathbf{x}^{\prime}\right|}{l_{G}}\right)^{2}\right]$

where $l_{G}$ denotes the correlation length. Again, the correlation length is chosen such that the reference error is close to $5 \%$. For a correlation length $l_{G}=0.77$, the reference error $\varepsilon_{V a r, \text { ref }}$ was obtained numerically as 0.049931 using the FCM with $4 \times 4$ cells and a maximum polynomial order of 15 . Special care was taken to ensure a good quality of the numerical integrals involved.

For the Gaussian correlation structure, the time needed to obtain an approximation of the random field for given relative errors is depicted in Figure 7. Comparing Figure 7 with Figure 5 for the exponential correlation structure, the main difference is in the efficiency of the Nyström (EOLE) method with respect to the FEM. For the case with an exponential correlation structure the two method behave similarly, and for the case with the Gaussian correlation structure the Nyström (EOLE) method is clearly more efficient than the FEM. This is because the Nyström method approximates the eigenfunctions using the kernel $\operatorname{Cov}\left(\mathbf{x}, \mathbf{x}^{\prime}\right)$. If the kernel is not differentiable, the theoretically smooth eigenfunctions are approximated by a linear combination of non-differentiable functions.

\section{CONCLUSIONS}

This paper assessed methods for the numerical solution of the integral eigenvalue problem in the 
Karhunen-Loève (KL) expansion. The methods were compared in terms of the computational costs of obtaining a random field approximation and of evaluating a realization of the random field.

The advantage of the Nyström method is its efficiency in obtaining a random field approximation. Moreover, the EOLE method, as a special case of the Nyström method that utilizes equal integration weights, is straightforward to implement. The reason is: the assembly of the matrix eigenvalue problem does not require an integration. This is contrary to the Galerkin-based approaches, where the coefficients of the matrix eigenvalue problem are obtained from a two-folded integration over the domain of the random field.

The advantage of the Galerkin-based procedures is that realizations of the random field can be evaluated faster than with the Nyström method. In this regard, the finite element method (FEM) and the finite cell method (FCM) outperform the Nyström method; where the finite element method (FEM) is faster than the finite cell method (FCM). Therefore, the Galerkinbased procedures are of interest if the computation of random field realizations constitutes a major part of the overall runtime; e.g. in reliability analysis. A linear projection of the covariance kernel in the FEM was shown to be less efficient than the standard $L^{2}$ projection. If a finite element mesh is not readily available and time-consuming to generate, the FCM is a feasible alternative that does not require an explicit meshing of the domain.

We recommend to use the FEM or the FCM only if a large number of realizations of the random field is required, and if the aim is to provide a reusable implementation as part of a library or software. In all other cases, we recommend to use the EOLE method; mainly because it is straightforward to implement.

\section{ACKNOWLEDGMENTS}

With the support of the Technische Universität München - Institute for Advanced Study, funded by the German Excellence Initiative.

\section{REFERENCES}

Atkinson, K. (1997). The numerical solution of integral equations of the second kind, Volume 4. Cambridge, UK: Cambridge University Press.

Betz, W., I. Papaioannou, \& D. Straub (2013). Numerical methods for the discretization of random fields by means of the Karhunen-Loève expansion. Manuscript submitted to Comput. Methods Appl. Mech. Engrg.

Düster, A., J. Parvizian, Z. Yang, \& E. Rank (2008). The finite cell method for three-dimensional problems of solid mechanics. Comput. Methods Appl. Mech. Engrg. (197), 3768-3782.

Ghanem, R.-G. \& P.-D. Spanos (1991). Stochastic Finite Elements - A Spectral Approach. Berlin: Springer.

Grigoriu, M. (1984). Crossing of non-Gaussian translation processes. Journal of Engineering Mechanics 110(41), 610-620.
Keese, A. (2004). A general purpose framework for stochastic finite elements. Ph. D. thesis, TU Braunschweig.

Li, C.-C. \& A. Der Kiureghian (1993, June). Optimal Discretization of Random Fields. Journal of Engineering Mechanics 119(6), 1136-1154.

Loève, M. (1977). Probability theory. 1977. Berlin: SpringerVerlag.

Papaioannou, I. (2013). Non-intrusive finite element reliability analysis. Saarbrücken: Südwestdeutscher Verlag für Hochschulschriften.

Parvizian, J., A. Düster, \& E. Rank (2007). Finite cell method. Computational Mechanics (41), 121-133.

Sudret, B. \& A. Der Kiureghian (2000, November). Stochastic Finite Element Methods and Reliability - A State-of-theArt Report. Technical Report UCB/SEMM-2000/08, Department of Civil \& Environmental Engineering, Univ. of California, Berkeley.

Van Trees, H. (1968). Detection, Estimation, and Modulation: Part I. New York: Wiley. 